\title{
Basilar Artery Changes in Fabry Disease
}

\author{
(D) Ranara, (DR.Y. Carlier, (D). Righetto, (D). Citton, (D) G. Locatelli, (D). Colas, (DM. Ermani, DD.P. Germain, and (D)A. Burlina
}

\section{ABSTRACT}

BACKGROUND AND PURPOSE: Dolichoectasia of the basilar artery is a characteristic finding of Fabry disease. However, its prevalence, severity, and course have been poorly studied. This study quantitatively evaluated, by MRA, a panel of basilar artery parameters in a large cohort of patients with Fabry disease.

MATERIALS AND METHODS: Basilar artery mean diameter, curved length, "origin-to-end" linear distance (linear length), and tortuosity index ([curved length $\div$ linear length] - 1) were retrospectively measured on 1.5T MRA studies of 110 patients with Fabry disease (mean age, $39.4 \pm 18.6$ years; 40 males) and 108 control patients (mean age, $42.0 \pm 18.2$ years; 40 males).

RESULTS: Patients with Fabry disease had increased basilar artery mean diameter $(P<.001)$ and basilar artery linear length $(P=.02)$ compared with control patients. Basilar artery curved length and tortuosity index correlated with age in both groups $(P<.001)$, whereas basilar artery linear length correlated with age only in patients with Fabry disease $(P=.002)$. Patients with Fabry disease showed a basilar artery curved length mean increase of $4.2 \%$ ( $9.7 \%$ in male patients with Fabry disease versus male control patients), whereas the basilar artery mean diameter had a mean increase of $12.4 \%$ (14.3\% in male patients with Fabry disease versus male control patients). Male patients with Fabry disease had increased basilar artery mean diameter, curved length, and tortuosity index compared with female patients with Fabry disease $(P=.04, P=.02$, and $P<.001$, respectively) and male control patients $(P<.001, P=.01$, and $P=.006$, respectively). Female patients with Fabry disease demonstrated an age-dependent increase of basilar artery mean diameter that became significant $(P<.001)$ compared with female control patients above the age of 45 years.

CONCLUSIONS: The basilar artery of patients with FD is subjected to major remodeling that differs according to age and sex, thus providing interesting clues about the pathophysiology of cerebral vessels in Fabry disease.

ABBREVIATION: $F D=$ Fabry disease

abry disease (OMIM 301500; FD) is a rare X-linked (Xq22.1) disease caused by mutations in the GLA gene causing deficiency of the hydrolase $\alpha$-galactosidase A ( $\alpha$-GalA, E.C. 3.2.1.22). ${ }^{1}$ The enzyme deficiency results in impaired sphingo-

Received August 20, 2016; accepted October 6.

From the Sezione di Neuroscienze (R.M.), University of Salerno, Salerno, Italy; Radiology Division (R.Y.C., F.C.) and Division of Medical Genetics (F.C., D.P.G.), University of Versailles, Versailles, France; Department of Neurosciences (S.R., V.C., M.E.), University of Padova, Padova, Italy; S. Giovanni e Ruggi d'Aragona Hospital (G.L.),

Salerno, Italy; and Neurological Unit (A.B.), St. Bassiano Hospital, Bassano del Grappa, Italy.

D.P.G. and A.B. should be considered co-senior authors.

Please address correspondence to Alessandro Burlina, MD, PhD, Neurological Unit, St. Bassiano Hospital, Via dei Lotti 40, 36061 Bassano del Grappa, Italy; e-mail: alessandro.burlina@aslbassano.it

-- Indicates open access to non-subscribers at www.ajnr.org

三 Indicates article with supplemental on-line appendix and tables.

http://dx.doi.org/10.3174/ajnr.A5069 lipid catabolism with lysosomal accumulation of upstream metabolites (mainly globotriaosylceramide $\left[\mathrm{Gb}_{3}\right]$ and its deacylated compound globotriaosylsphingosine $\left[\operatorname{lysoGb}_{3}\right]$ ). All organs are involved, with major damage to the kidneys, heart, and nervous system. The brain might present white matter vascular-like abnormalities, TIAs, and stroke at a young age, ${ }^{2}$ suggesting that micro- and macroangiopathy might have a pivotal role in the pathogenesis of brain lesions. Indeed, both small and large blood vessels have been consistently shown to present functional and morphologic changes in patients with FD. ${ }^{3-5}$ Increased vessel tortuosity has been found in the retina ${ }^{6}$ and in the skin, ${ }^{7,8}$ and intracranial artery dolichoectatic changes have been repeatedly observed in patients with FD during both pathologic and neuroimaging evaluations. ${ }^{9-17}$

Hitherto, most of the latter studies either referred to small samples or evaluated single specific aspects of artery dolichoectasia (such as the vessel lumen diameter). In addition, these studies 


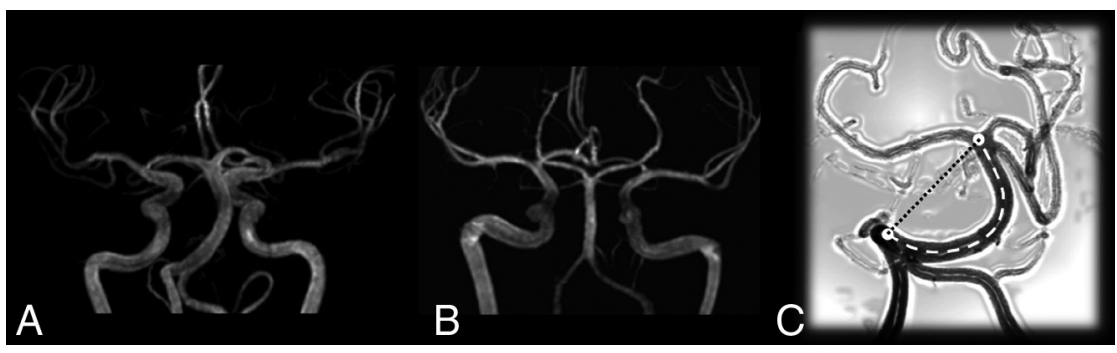

FIG 1. MIP of an MRA study in A, a 41-year-old male patient with FD and $B$, a control patient. Note a mild increase of the tortuosity of the basilar artery and a more evident increase of the lumen diameter (compare the basilar artery with the contiguous ICA) C, Drawing of a tortuous basilar artery showing the real curved length (dashed line) and the linear distance between the basilar artery extremes (dotted line).

applied different quantification methods or semiquantitative scores for the severity of the intracranial FD-related vessel changes, leading to conflicting results about the role of sex, age, and treatments.

Our retrospective, transversal, case-control MRA study quantitatively investigated the morphologic basilar artery lumen changes (Fig 1) by applying a comprehensive panel of measurements encompassing all aspects of vessel dolichoectasia (diameter, length, and tortuosity) on a large cohort of patients with FD, aiming to provide a detailed picture of FD-related basilar artery changes according to age and sex.

\section{MATERIALS AND METHODS \\ Patients}

One hundred thirteen patients with FD underwent MR imaging and MRA and were retrospectively analyzed. Three patients were excluded for anatomic reasons (see below): therefore, 110 patients with FD were considered (mean age, $39.4 \pm 18.6$ years; range, $6-75$ years; 40 males). The diagnosis of FD was confirmed by $\alpha$-galactosidase A enzyme activity and GLA mutation identification. All patients gave written informed consent.

One hundred ten patients with no history of head trauma, neurosurgery, or other neurologic or psychiatric disease who underwent MRI and MRA for headache or TIA were analyzed. Two patients were excluded for anatomic reasons (see below): therefore, 108 patients (mean age, $42.0 \pm 18.2$ years; range: $4-76$ years; 40 males) represented our control group.

\section{MR Technique}

All MR examinations of patients and control patients were performed between 2007-2014 with 1.5T scanners (Achieva or Intera; Philips Healthcare, Best, the Netherlands) with a standard quadrature head coil.

The MR study protocol included conventional T1-weighted and FLAIR axial images covering the whole brain and a TOFMRA sequence for the evaluation of the main intracranial arteries. The latter sequence was acquired with the following parameters: TR, $23 \mathrm{~ms}$; TE, $2.274 \mathrm{~ms}$; section thickness, $1.4 \mathrm{~mm}$; acquisition matrix, $512 \times 512$; and FOV, $16 \mathrm{~cm}$ on the Intera scanner and TR, $25 \mathrm{~ms}$; TE, $6.906 \mathrm{~ms}$; section thickness, $1 \mathrm{~mm}$; acquisition matrix, $512 \times 512$; and FOV, $16 \mathrm{~cm}$ on the Achieva scanner.

\section{Basilar Artery Evaluation}

All MRIs were evaluated by 2 neuroradiologists (R.M., V.C.) to exclude concomitant posterior cranial fossa lesions that could potentially interfere with the cisternal course of the basilar artery (eg, neoplasms, abscesses, and parenchymal or vascular malformations). No patient with FD or healthy patient was excluded for this reason, and in no case was there disagreement between neuroradiologists.

The mean basilar artery diameter was measured by a neuroradiologist (R.M.) on the acquisition MRA axial sections with a commercially available image viewer (Medstation version 4.9; Exprivia, Molfetta, Italy) at 3 points, namely the starting, middle, and ending points of the basilar artery after adjusting the image window (On-line Appendix).

The basilar artery anatomic length (curved length of the whole cisternal segment) and the linear distance between the proximal and distal extremities of the basilar artery (linear length) were measured by a neuroradiologist (S.R.) with commercially available software (InSpace, syngo MR Workplace; Siemens, Erlangen, Germany) dedicated to the vessel analysis (On-line Appendix). Because the basilar artery landmarks were 1) the conjunction of the 2 vertebral arteries (starting point) and 2) the basilar artery bifurcation into the 2 posterior cerebral arteries (ending point), patients with FD and control patients with agenesia of 1 vertebral artery or of the precommunicating segment of 1 or both posterior cerebral arteries were excluded from the study ( 2 control patients and 3 patients with FD, including a woman harboring a persistent trigeminal artery).

In addition, the tortuosity index was calculated as follows, according to previous studies ${ }^{18}$ :

$$
\text { Tortuosity Index }=(\text { curved length } \div \text { linear length })-1
$$

This index is equal to 0 when the vessel is perfectly straight, and the index increases with the relative increase of the curved length with respect to the linear length (Fig 1C).

In 15 patients, all of the aforementioned variables were remeasured after 3 months by 2 of the coauthors (R.M., S.R.) to assess intrarater and interrater reproducibility.

\section{Statistical Analysis}

The Student $t$ test was performed to compare quantitative normally distributed variables between patients with FD and control patients and between subgroups. Analysis of covariance was used to test the dependence of groups, omitting the effect of age. The linear relationships between age and diameter, length, and tortuosity measures were evaluated by using the Pearson coefficient. Reproducibility of the measurements was also tested by Pearson coefficient. Significance level was set at $P<.05$.

\section{RESULTS}

Main findings are presented in On-line Table 1. FD and control groups did not differ for age $(P=.30)$ or sex $(P=.91)$. All measurements (mean diameter, curved length, linear length, and tortuosity index) showed very high interrater and intrarater agreement $(r \geq 0.9)$. 
Controls
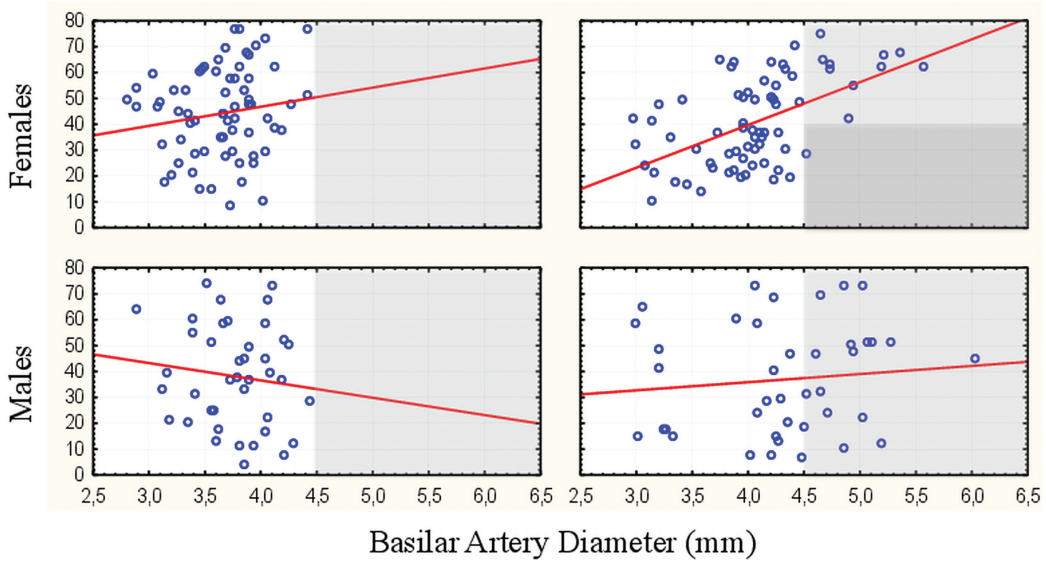

FIG 2. Mean basilar artery diameter findings in our study population (patients with FD and control patients) subdivided according to sex (circles represent the value in each patient). This composite plot shows sex- and age-related differences among subgroups; note that a mean basilar artery diameter greater than $4.5 \mathrm{~mm}$ could be found at any age among male Fabry patients and almost only after the age of 40 among female Fabry patients.

\section{Diameter Analysis}

Compared with control patients, patients with FD had increased mean basilar artery diameter $(4.16 \pm 0.62 \mathrm{~mm}$ versus $3.70 \pm 0.36$ $\mathrm{mm} ; P<.001)$; the mean basilar artery diameter showed mild correlation with age among patients with $\mathrm{FD}(r=0.3 ; P=.001)$ and no correlation among control patients.

When subgrouping according to sex, the basilar artery diameter did not correlate with age among male patients with FD and male and female control patients. In contrast, female patients with FD showed a good correlation between age and basilar artery diameter $(r=0.54)$ (Fig 2).

Male patients with FD had increased mean basilar artery diameter compared with female patients with FD $(4.32 \pm 0.72 \mathrm{~mm}$ versus $4.07 \pm 0.55 \mathrm{~mm} ; P=.04)$ and male control patients $(4.32 \pm 0.72 \mathrm{~mm}$ versus $3.78 \pm 0.36 \mathrm{~mm} ; P<.001)$. Female patients with FD had increased mean basilar artery diameter compared with female control patients $(4.07 \pm 0.55$ mm versus $3.66 \pm$ $0.36 \mathrm{~mm} ; P<.001)$. No difference was found between male and female control patients $(3.70 \pm 0.36 \mathrm{~mm}$ versus $3.66 \pm 0.36 \mathrm{~mm})$.

We divided patients and control patients according to sex and age (younger or older than 45 years); male patients with FD showed increased mean basilar artery diameter compared with control patients at any age, whereas female patients with FD differed from control patients only in the older subgroup.

A diagnostic criterion based on a mean basilar artery diameter $>4.42 \mathrm{~mm}$ had a specificity of $97.2 \%$ and a sensitivity of $28.2 \%$.

\section{Length and Tortuosity Analysis}

Compared with control patients, patients with FD had increased linear length ( $28.34 \pm 4.03 \mathrm{~mm}$ versus $27.18 \pm 3.32 \mathrm{~mm} ; P=.02)$ and showed a trend toward increased curved length $(31.13 \pm 5.38$ $\mathrm{mm}$ versus $29.88 \pm 4.79 \mathrm{~mm} ; P=.07)$. As a consequence, the tortuosity index did not differ between patients with FD and control patients $(0.097 \pm 0.083$ versus $0.099 \pm 0.113 ; P=.86)$. Curved length and tortuosity index showed a positive correlation with age in both the patient and control groups $(P<.001)$. In contrast, the linear length correlated with age only in the FD group $(P=$ $.002)$.

Male patients with FD had increased curved length and tortuosity index compared with female patients with FD $(32.72 \pm 5.81 \mathrm{~mm}$ versus $30.21 \pm 4.92$ $\mathrm{mm} ; P=.02$ and $0.132 \pm 0.094$ versus $0.077 \pm 0.070 ; P<.001$, respectively) and male control patients $(32.72 \pm 5.81$ mm versus $29.83 \pm 4.5 \mathrm{~mm} ; P=.01$ and $0.132 \pm 0.094$ versus $0.075 \pm 0.085 ; P=$ $.006)$. No significant difference was observed between male and female control patients. No significant difference was found between female patients with FD and female control patients after adjusting for age.

\section{Analysis of Diameter Versus Length and Tortuosity}

The mean basilar artery diameter showed no correlation with curved length and tortuosity index in control patients and showed a mild correlation $(r=0.35$ and $r=0.43$, respectively) in patients with $\mathrm{FD}$.

The curved length showed a mean increase of $4.2 \%$ in patients with FD compared with control patients $(9.7 \%$ in male patients with FD compared with male control patients), whereas the mean lumen diameter (ie, circumference) showed a mean increase of $12.4 \%$ in patients with FD compared with control patients (14.3\% in male patients with FD compared with male control patients).

\section{DISCUSSION}

This MRA study quantitatively investigated the morphologic basilar artery lumen changes in FD, showing the progressive and profound vessel remodeling that ultimately leads to basilar artery dolichoectasia. In particular, patients with FD presented increased basilar artery diameter, length, and tortuosity that differed significantly according to patient sex and age.

\section{Basilar Artery Diameter Changes}

Ectasia of intracranial arteries has been qualitatively observed in patients with FD at both pathologic and neuroimaging evaluation. ${ }^{9-13}$ A few studies applied a semiquantitative or quantitative approach with MRA 3D reconstructions (2 studies with $1.5 \mathrm{~T}$ and 1 with 3T scanner), ${ }^{14-16,19}$ T2 axial images (van der Tol L, personal communication), either T2 axial images or MRA, ${ }^{17}$ or axial partitions of MRA (present study). Despite different methods, sequences, and scanners used for the measurement and the different ways to present study findings (different patient subgrouping, mean or median value, etc), all studies consistently showed the increase of the basilar artery diameter in patients with FD compared with control patients (On-line Table 2).

As expected for an X-linked disease, the basilar artery diameter in FD was shown to be significantly greater among male patients compared with female patients in all previous studies except one. ${ }^{19}$ The latter discordant result might have depended on the 
small sample size (5 males versus 7 females) or on the age of the sex subgroups considered in the study (mean age of female study participants, $>40$ years). In fact, according to our findings, the basilar artery diameter appears to already be enlarged in male patients with FD at a young age, whereas women show significant basilar artery changes only above the age of 45 years. The different behavior of basilar artery diameter according to sex is interesting for several reasons. For example, it limits the efficacy of basilar artery diameter as a criterion for differentiating female patients with FD from patients with multiple sclerosis, as recently suggested. ${ }^{20}$ In fact, the diagnostic dilemma usually involves young women aged less than 45 years with neurologic symptoms and nonspecific white matter signal abnormalities. ${ }^{20}$ In addition, because WM changes also seem to appear early in female patients with FD, but basilar artery changes occur later in women, macroangiopathy seems to have no role or a minimal role in the appearance of white matter signal abnormalities. Therefore, micro- and macroangiopathy might present different time courses in FD.

\section{Length and Tortuosity of the Basilar Artery}

The approach applied in this study provides quantitative information on the effect of FD on the elongation of the basilar artery. Indirect data have been provided by previous case reports, case series, and small studies based on qualitative evaluations (On-line Table 3). ${ }^{9,10,13}$ Recently, Politei et al ${ }^{17}$ applied a semiquantitative approach with the Smoker criteria. According to the Smoker criteria, in addition to a basilar diameter greater than $4.5 \mathrm{~mm}$, the position of the top of the basilar artery (indicative of the length of the artery) and the basilar artery position with respect to the midline (indicative of the tortuosity of the artery) also concur in the identification of dolichoectasic basilar arteries. Politei et $\mathrm{al}^{17}$ found an increased rate of dolichoectasia in patients with FD, especially among males (55.5\% of male patients with FD versus $34.8 \%$ of female patients with FD; $P=.09$ ). Unfortunately, this study did not include a control population and did not provide the weight of each item (diameter, elongation, and tortuosity) in determining dolichoectasia in the FD population, hampering their precise analysis.

In the present study, FD-related vessel elongation was directly demonstrated by the increased curved length (effective length of the basilar artery in its 3D cisternal course) in male patients with FD compared with female patients with FD and control patients. In addition, patients with FD showed increased linear distance between the proximal and distal extremities of the basilar artery. The concomitant cranial displacement of the top of the basilar artery likely determines a higher score at the second item of the Smoker criteria, thus contributing to the high rate of dolichoectasia observed in patients with FD. ${ }^{17}$ The measurement of both curved and linear basilar artery length allowed us to precisely quantify the basilar artery tortuosity by a tortuosity index. Although the concomitant increase of the linear length partly hampered the efficacy in detecting basilar artery tortuosity changes, this measure further highlighted the sex-related artery vulnerability in FD, showing a significantly increased basilar artery tortuosity in male patients with FD compared with both female patients with FD and male control patients. Although the Smoker score is easily applicable to conventional T2 axial images and is certainly less time-consuming, the quantitative evaluation of linear length, curved length, and tortuosity index on 3D-TOF MRA proved to be highly reproducible in our study, providing a powerful and reliable tool for the follow-up of the natural history of artery changes in FD over the years as well as the monitoring of any therapeutic intervention.

\section{Evolution of Basilar Artery Changes}

The evolution of basilar artery changes is an obvious phenomenon that can be inferred by the fact that megadolicho-arteries do not appear, or at least have never been reported, in pediatric patients with FD. Nonetheless, the characterization of the evolution of basilar artery changes in FD remains rather elusive. Azevedo et $\mathrm{al}^{19}$ found increased basilar artery diameter values in older patients with FD, whereas Fellgiebel et $\mathrm{al}^{14,15}$ and Uçeyler et $\mathrm{al}^{16}$ found no association with age. The present study showed that the basilar artery diameter increases significantly with age in the female subgroup, whereas male patients with FD and both male and female control patients do not change significantly over time. According to these findings, male patients with FD already present increased mean basilar artery diameter at a young age, with scarce changes over time, whereas female patients with FD progress from values that do not differ from control patients to values that do not differ from male patients with FD. Altogether, these contradictory findings underlie 1) the heterogeneity of basilar artery involvement in FD and 2) the difficulty in extrapolating complex phenomena in rare diseases without well-designed, long-lasting longitudinal studies in large patient cohorts with sex- and agematched control patients. For example, the lack of evolution of basilar artery diameter in men might be related to a higher early mortality in patients with more severe intracranial artery involvement. Because stroke is associated with an increased basilar artery diameter in the healthy population ${ }^{21}$ and stroke occurs at a younger age in male patients with $\mathrm{FD},{ }^{2}$ a biased selection might have altered basilar artery diameter age-dependent findings in a transverse study design.

Hitherto, the evolution of basilar artery elongation has never been addressed, and all the information can be driven only from the present study, which necessarily requires further validation. According to our findings, basilar artery elongation seems to be an age-dependent phenomenon recognizable in both patients with FD and control patients. Nonetheless, patients with FD also present an age-dependent significant increase of the linear distance between the proximal and distal extremities of the basilar artery, which likely results in cranial ectopia of its distal extremity.

Altogether, these artery changes involving diameter, length, and tortuosity might explain the high rate of dolichoectasia previously detected semiquantitatively in patients with FD. ${ }^{17}$

\section{Ectasia Versus Elongation}

Dolichoectatic changes might represent different faces of the same phenomenon (ie, the structural disruption of the artery vessel wall) or underlie different pathogenic mechanisms. Actually, 
diameter, curved length, and tortuosity index showed no correlation among control patients and displayed a relatively weak relationship within the FD cohort. In addition, the severity of the curved length and diameter changes in the FD cohort compared with control patients demonstrates that the artery wall is nearly 3 times more vulnerable circumferentially than longitudinally. This observation seems to be consistent with functional and pathologic findings in FD that report the primary involvement of the smooth muscle cells in the accumulation of globotriaosylceramide, reduced sympathetic innervation of proximal cerebral arteries, and enhanced release of nitric oxide from endothelial cells. ${ }^{5}$ Because the smooth muscle cell tone is pivotal in controlling the vessel radius according to brain parenchyma metabolic need, media layer dysfunction might manifest prevalently as ectasia and, only to a lesser extent, elongation. The presence of basilar artery ectasia in other lysosomal storage disorders, like late-onset Pompe disease, might support the role of metabolite accumulation within the smooth muscle cells. ${ }^{22,23}$

\section{Diagnostic Role of Basilar Artery Changes in FD}

Limits inherent to the measurement of the basilar artery diameter and to scanners, sequences, and methods applied have been discussed above. For example, the evaluation of the basilar artery diameter on MRA most likely leads to an underestimation compared with T2 axial findings because MRA represents only the vessel lumen, whereas T2 imaging measurement includes the vessel wall. In addition, sequences with long TR and TE (T2 imaging) are prone to CSF pulsation artifacts around the basilar artery that might alter the measurement of the vessel diameter. Sequences on different scanners might differ for resolution, with a considerable impact on the measurement of the basilar artery diameter. Nonetheless, at least 2 further anatomic and methodologic aspects need to be discussed. In contrast to the internal carotid artery, the basilar artery shows high diameter variability even among patients who do not have FD $(2.8-4.4 \mathrm{~mm}$ in the present study, but also $1.2-5.5 \mathrm{~mm}) .{ }^{16}$ The caliper variability of the vertebral arteries and the rather common variants of the circle of Willis involving the precommunicating P1 segment of the posterior cerebral arteries might significantly influence the basilar artery diameter, hampering its diagnostic value. In fact, nearly $20 \%$ of P1 segments are hypoplastic, and the blood flow of the postcommunicating P2 segment might be partly or completely sustained by the ipsilateral internal carotid artery via the posterior communicating artery. A significant basilar artery diameter increment in a patient with FD with unilateral or bilateral P1 segment hypoplasia would probably not lead to "increased" values compared with the normal mean value. This anatomic variability might result in a decreased sensitivity of basilar artery diameter cutoff values when looking for a high specificity (a cutoff of $4.42 \mathrm{~mm}$ in the present study had a specificity of $97.2 \%$, but a sensitivity of $28.1 \%$ ), especially among young women, in whom the differences between patients and control patients are less evident, if there are any.

The methodologic aspect regards the variability of the diameter according to image windowing. Previous studies did not specify the window parameters used for the evaluation of the basilar artery diameter because they depend on the investigator's choice and on image features that might vary from patient to patient, even when using the same sequence parameters. Different image windowing might lead to the inclusion or exclusion of a pixel at the border of the vessel. Because the pixel size is usually $0.3-0.4$ $\mathrm{mm}$ and the mean basilar artery diameter in control patients is approximately $3.7 \mathrm{~mm}$, different image windowing might lead to a $10 \%-20 \%$ variability in the measure obtained. This fact might partly explain the difficulty of replicating cutoff values in different studies. A previous study investigated 25 patients with FD and showed that a basilar artery diameter cutoff of $2.98 \mathrm{~mm}$ could differentiate patients with FD from young patients without FD who have had a stroke with a specificity of $88.5 \%$ and a sensitivity of $84 \% .{ }^{15}$ Another study on 87 patients with FD demonstrated that a basilar artery diameter cutoff of $3.2 \mathrm{~mm}$ had a sensitivity of $87 \%$ and a specificity of $86 \%$ in discriminating male patients with FD from male control patients (no difference was found between male patients with FD and male patients without FD who have had a stroke or among female subgroups). ${ }^{16}$

Therefore, to minimize the measurement variability, we applied an easily replicable standardized method to optimize and standardize the image windowing throughout the study (On-line Appendix). Indeed, we obtained outstanding interrater and intrarater reproducibility of the basilar artery diameter measurements. Nonetheless, because differences in image windowing, sequences, sequence parameters, and scanners might significantly impact the final measure of the vessel diameter, center-specific normative values are likely required, and literature cutoff values can hardly be used in a diagnostic setting of FD. Taking into account the anatomic variability of the circle of Willis, the use of the basilar artery diameter as a unique marker of FD appears rather inefficient.

Similarly, basilar artery linear and curved length and the relative tortuosity index proved to retain very poor diagnostic utility because of the largely overlapping findings between patients with FD and control patients. These limits are minimized in a longitudinal study design because the anatomy of the circle of Willis does not change significantly over time, thus allowing precise monitoring of the basilar artery dolichoectatic changes.

To note, the inclusion of patients with headache and TIA in our control group might represent a possible drawback of the study. Even though no study has shown basilar artery changes in patients with headache and TIA, these patients may potentially present initial dolichoectatic abnormalities that might have hampered the recognition of FD basilar artery changes. According to this hypothesis, basilar artery changes in FD may be even more evident when using healthy patients as control patients. However, a positive control group has the advantage of being closer to a clinical setting, where some of the abovementioned vascular changes have been proposed for diagnostic purposes.

\section{CONCLUSIONS}

The panel of measurements (artery diameter, length, and tortuosity) applied in this study provides some relevant hints about the pathophysiology of vessel remodeling in FD. Moreover, our study provides an interesting tool for monitoring the natural course of 
basilar artery changes and the possible response to treatments. Despite these findings, basilar artery measures seem to retain limited value in a routine diagnostic setting unless a highly selective basilar artery diameter cutoff is considered.

Disclosures: Renzo Manara—UNRELATED: Payment for Lectures (including service on Speakers Bureaus): Biomarin, Merks, Shire, Comments: honoraria for lectures. Giampiero Locatelli-UNRELATED: Travel/Accommodations/Meeting Expenses Unrelated to Activities Listed:, Comments: Boston paid fees for congresses and courses about interventional neuroradiology (approximately $€ 700$ per course or congress). Dominique Germain—UNRELATED: Consultancy: Sanofi-Genzyme, Shire; Grants/Grants Pending: Amicus, Sanofi-Genzyme, Shire*; Payment for Lectures (including service on Speakers Bureaus): Sanofi-Genzyme, Shire; Travel/Accommodations/Meeting Expenses Unrelated to Activities Listed: Sanofi-Genzyme, Shire. Alessandro Burlina-UNRELATED: Board Membership: Sanofi-Genzyme, Comments: approximately $€ 2000$ as member of the European Advisory Board of the Fabry Registry, which is sponsored by Sanofi-Genzyme; Payment for Lectures (Including Service on Speakers Bureaus):, Comments: approximately €3000; Travel/Accommodations/Meeting Expenses Unrelated to Activities Listed:, Comments: related to the payment of lectures or seminars. ${ }^{*}$ Money paid to institution.

\section{REFERENCES}

1. Germain DP. Fabry disease. Orphanet J Rare Dis 2010;5:30 CrossRef Medline

2. Kolodny E, Fellgiebel A, Hilz MJ, et al. Cerebrovascular involvement in Fabry disease: current status of knowledge. Stroke 2015;46: 302-13 CrossRef Medline

3. Boutouyrie P, Laurent S, Laloux B, et al. Non-invasive evaluation of arterial involvement in patients affected with Fabry disease. $J$ Med Genet 2001;38:629-31 CrossRef Medline

4. Boutouyrie $\mathrm{P}$, Laurent $\mathrm{S}$, Laloux B, et al. Arterial remodelling in Fabry disease. Acta Paediatr Suppl 2002;91:62-66 CrossRef Medline

5. Rombach SM, Twickler TB, Aerts JM, et al. Vasculopathy in patients with Fabry disease: current controversies and research directions. Mol Genet Metab 2010;99:99-108 CrossRef Medline

6. Orssaud C. Ocular manifestations in Fabry disease: a survey of $\mathbf{3 2}$ hemizygous male patients. Ophthalmic Genet 2003;24:129-39 CrossRef Medline

7. Zampetti A, Gnarra M, Borsini W, et al. Vascular endothelial growth factor (VEGF-a) in Fabry disease: association with cutaneous and systemic manifestations with vascular involvement. Cytokine 2013; 61:933-39 CrossRef Medline

8. Michaud L. Vascular tortuosities of the upper eyelid: a new clinical finding in Fabry patient screening. J Ophthalmol 2013;2013:207573 CrossRef Medline

9. Garzuly F, Maródi L, Erdös M, et al. Megadolichobasilar anomaly with thrombosis in a family with Fabry's disease and a novel muta- tion in the alpha-galactosidase A gene. Brain 2005;128:2078-83 CrossRef Medline

10. Maisey DN, Cosh JA. Basilar artery aneurysm and AndersonFabry disease. J Neurol Neurosurg Psychiatry 1980;43:85-87 CrossRef Medline

11. Jardim L, Vedolin L, Schwartz IV, et al. CNS involvement in Fabry disease: clinical and imaging studies before and after 12 months of enzyme replacement therapy. J Inherit Metab Dis 2004;27:229-40 CrossRef Medline

12. Gupta S, Ries M, Kotsopoulos S, et al. The relationship of vascular glycolipid storage to clinical manifestations of Fabry disease: a cross-sectional study of a large cohort of clinically affected heterozygous women. Medicine (Baltimore) 2005;84:261-68 CrossRef Medline

13. Ginsberg L, Manara R, Valentine AR, et al. Magnetic resonance imaging changes in Fabry disease. Acta Paediatr Suppl 2006;95:57-62 CrossRef Medline

14. Fellgiebel A, Keller I, Marin D, et al. Diagnostic utility of different MRI and MR angiography measures in Fabry disease. Neurology 2009;72:63-68 CrossRef Medline

15. Fellgiebel A, Keller I, Martus $\mathrm{P}$, et al. Basilar artery diameter is a potential screening tool for Fabry disease in young stroke patients. Cerebrovasc Dis 2011;31:294-99 CrossRef Medline

16. Uçeyler N, Homola GA, Guerrero González H, et al. Increased arterial diameters in the posterior cerebral circulation in men with Fabry disease. PLoS One 2014;9:e87054 CrossRef Medline

17. Politei J, Schenone AB, Burlina A, et al. Vertebrobasilar dolichoectasia in Fabry disease: the earliest marker of neurovascular involvement? J Inborn Errors Metab Screen 2014; DOI: 10.1177/2326409814541246

18. Manara R, Gabrieli J, Citton V, et al. Intracranial internal carotid artery changes in acromegaly: a quantitative magnetic resonance angiography study. Pituitary 2014;17:414-22 CrossRef Medline

19. Azevedo E, Mendes A, Seixas D, et al. Functional transcranial Doppler: presymptomatic changes in Fabry disease. Eur Neurol 2012;67:331-37 CrossRef Medline

20. Böttcher T, Rolfs A, Tanislav C, et al. Fabry disease - underestimated in the differential diagnosis of multiple sclerosis? PLoS One 2013;8: e71894 CrossRef Medline

21. Pico F, Labreuche J, Gourfinkel-An I, et al. Basilar artery diameter and 5-year mortality in patients with stroke. Stroke 2006;37: 2342-47 CrossRef Medline

22. Sacconi S, Bocquet JD, Chanalet S, et al. Abnormalities of cerebral arteries are frequent in patients with late-onset Pompe disease. J Neurol 2010;257:1730-33 CrossRef Medline

23. Laforêt $\mathrm{P}$, Petiot $\mathrm{P}, \mathrm{Nicolino} \mathrm{M}$, et al. Dilative arteriopathy and basilar artery dolichoectasia complicating late-onset Pompe disease. Neurology 2008;27:70:2063-66 CrossRef Medline 